\title{
DETECCIÓN Y CARACTERÍSTICAS DE CÉLULAS PROSTÁTICAS CIRCULANTES PRIMARIAS, ASOCIACIÓN CON LA PRESENCIA DE MICROMETÁSTASIS Y LAS IMPLICACIONES PARA EL TRATAMIENTO QUIRÚRGICO EN HOMBRES CON CÁNCER PROSTÁTICO
}

Nigel P. Murray 1,2,3, Eduardo Reyes5, Leonardo Badínez4, Nelson Orellana ${ }^{7}$, Ricardo Dueñas ${ }^{7}$ y Cinthia Fuentealba ${ }^{5}$.

'Hospital de Carabineros de Chile. Santiago de Chile.

2Instituto de BioOncología, Santiago de Chile.

${ }^{3}$ Facultad de Medicina. Universidad Mayor. Santiago de Chile.

4Fundación Arturo López Pérez, Santiago de Chile.

${ }^{5}$ Becado Urología Universidad de Santiago. Santiago de Chile.

\begin{abstract}
Resumen.- OBJETIVO: Determinar la frecuencia de céIulas prostáticas circulantes (CPCs) primaria en hombres con cáncer de próstata en el momento del diagnóstico, la asociación con micrometástasis ósea y subclasificación por CD82. Determinar la relación con la estadio patológica y la eficacia para seleccionar pacientes para la prostatectomía radical.
\end{abstract}

MÉTODOS: Se incluyeron hombres con diagnóstico de cáncer de próstata previo a tratamiento definitivo. Se obtuvieron muestras de sangre y de médula ósea, las células mononucleares separadas por centrifugación diferencial y células prostáticas identificadas con inmumocitoquímica con anti-APE, las muestras positivas fueron

\section{CORRESPONDENCIA}

Nigel P Murray

Sección de Hematología

Hospital de Carabineros de Chile

Simón Bolívar 2200, Ñuñoa.

Santiago de Chile.

nigelpetermurray@gmail.com

Aceptado para publicar: 29 de enero 2010 sub-clasificadas con anti-CD82. Se registraron también los detalles de APE sérico, Índice de Gleason y estadio patológica.

RESULTADOS: De 77 hombres, 58 (75,3\%) tuvieron $7^{\circ}$ CPCs detectadas. Hubo una asociación con estadio pero no con el Índice de Gleason, 37 (40,3\%) tuvieron micrometástasis. Hubo una asociación significativa con la estadio patológica y Índice de Gleason. Pacientes CPC negativa tuvieron una menor frecuencia de micrometástasis que los hombres CPC positiva 1/19 versus 30/58 ( $p<0,0003)$.

Hubo una relación inversa significativa entre la expresión de CD82 en CPCs y el índice de Gleason y menor frecuencia de micrometástasis en comparación con hombres CPC CD82 positivos ( $p<0,0005)$.

En el grupo de combinación de hombres CPC negativa y CPC positiva CD82 positivo la frecuencia de micrometastasis fue significativamente menor que el grupo CPC (+) CD82 (-) 5/39 versus 26/38 respectivamente/ $p<0$ ,0000007), con una sensibilidad de $87 \%$ y especificidad de 73,9\% para la ausencia de micrometástasis.

CONCLUSIONES: La presencia de CPCs implica un riesgo mayor de desarrollar micrometástasis, la co-expresión del CD82 es asociada con tumores de bajo grado, un riesgo disminuido del desarrollo de micrometástasis óseas. Como consecuencia, el uso de la detección de CPCs primarias y su sub-clasificación podrían ser clínicamente útiles para identificar los pacientes los cuales beneficiarán de una prostatectomía radical como tratamiento de primera línea. 
Palabras clave: Cáncer de próstata. Células prostáticas circulantes. Micrometástasis. CD82.

Summary.- OBJECTIVES: To determine the frequency of primary circulating prostate cells in men with prostate cancer at the time of diagnosis, the association with micrometastasis, sub-classification for CD82 and the relation with pathological stage. To determine their clinical usefulness to identify patients in whom radical prostatectomy would be first choice therapy.

METHODS: Men with the diagnosis of prostate cancer before definitive therapy. Blood and bone marrow samples were taken, mononuclear cells separated by differential centrifugation and prostate cells identified with immunocyłochemistry using anti-PSA. Positive samples were sub-classified with anti-CD82. Details of serum PSA, Gleason score and pathological stage were registered.

RESULTS: Of 77 men 58 (75.3\%) had primary CPCs detected, there was an association with stage but not Gleason. 31 (40.3\%) had micrometastasis with an association with stage and Gleason score. CPC-negative patients had fewer micrometastasis detected, 1/19 versus 30/58 ( $p<0.003)$.

There was an inverse relation between CD82 expression and Gleason score, men with CPCs expressing CD82 had fewer micrometastasis. The combined group of CPC negative and CPC positive CD82 positive men showed a sensitivity of $87 \%$ and specificity of $73.9 \%$ for the absence of micrometastasis.

CONCLUSIONS: The detection of CPCs and sub-classification with CD82 could be clinically useful to identify men with a significantly lower risk of micrometastais and as a consequence to identify men in whom radical prostatectomy could be the best initial treatment.

Keywords: Prostate cancer. Circulating tumor cells. Micrometastasis. CD82.

\section{INTRODUCCIÓN}

La determinación del nivel sérico de antígeno prostático específico en asociación al tacto rectal es considerada en la actualidad el estudio recomendado en el diagnóstico precoz del cáncer prostático (1). Esta ha permitido que un creciente número de pacientes sean diagnosticados en estadíos clínicos iniciales. Para el cáncer prostático localizado, la prostatectomía radical es considerado el tratamiento más adecuado $(2,3)$. Sin embargo, los hallazgos patológicos post-quirúrgicos (6) muestran que la proporción de cánceres potencialmente curados sólo con cirugía son entre el $40 \%$ y $70 \%$ (4), un $30 \%$ a $60 \%$ de los pacientes son patológicamente estadio pT3 (5) y un $20-70 \%$ tienen márgenes positivos.

Estos dos factores, estadio patológico y márgenes positivos, influyen de forma directa en la supervivencia libre de progresión bioquímica, recidiva local y sistémica (7). Con las limitaciones, como exámenes predictivos del estadio patológico, del estadio clínico, índice de Gleason, APE sérico, Partin et al (8) propusieron su análisis combinado para producir un nomograma para predecir el estadio patológico. Sin embargo, a pesar de todos los métodos utilizados para estadiaje clínico, la mitad de los pacientes son infraestadiados (5), lo que supone que entre un 17 $70 \%$ presente afectación extracapsular o márgenes afectados (6). Sabemos que la penetración extra-capsular o márgenes positivos son factores de directa influencia en la aparición de recurrencia local o a distancia (7). El alto porcentaje de infraestadiaje y márgenes positivos (17-70\%) (6) es una de las razones por las que, en los últimos tiempos, ha disminuido el entusiasmo por la prostatectomía radical (9), y ha motivado la exploración de caminos que mejoren la curabilidad quirúrgica.

La detección de células prostáticas en sangre (CPCs) y su papel en el desarrollo de metástasis continúa siendo controvertido. Publicaciones previas sugieren su utilidad en la orientación del manejo del cáncer de próstata (10). Estudios publicados en Chile han detectado la presencia de CPCs y la expresión de la proteína P504S en estadios precoces del cáncer de próstata, así como la relación entre presencia de CPCs en sangre y médula ósea con los factores de riesgo establecidos $(11,12)$.

Existen dos tipos de CPCs, las primarias (1을 CPCs) detectadas al tiempo del diagnóstico, y las secundarias $\left(2^{\circ} \mathrm{CPCs}\right)$ detectadas después de la terapia radical. Las características de las $1^{\circ} \mathrm{CPCs}$, como su presencia o ausencia, podrían ser importantes para determinar un cáncer localizado y por lo tanto apto para la cirugía o avanzado con la presencia de micrometástasis en la médula ósea, donde un tratamiento puramente quirúrgico será inadecuado.

El gen supresor tumoral KAil es ubicado en la región p 11.2 del cromosoma 11 (13) y se codifica para una glicoproteína de la familia de las tetraspaninas, el CD82. Tejidos prostáticos benignos, como la hiperplasia expresan el CD82 (13), la expresión es aumentada en hiperplasia benigna asociada con cáncer de bajo grado, como en cáncer prostático con un índice de Gleason de 3 y 4 (14), pero la ex- 

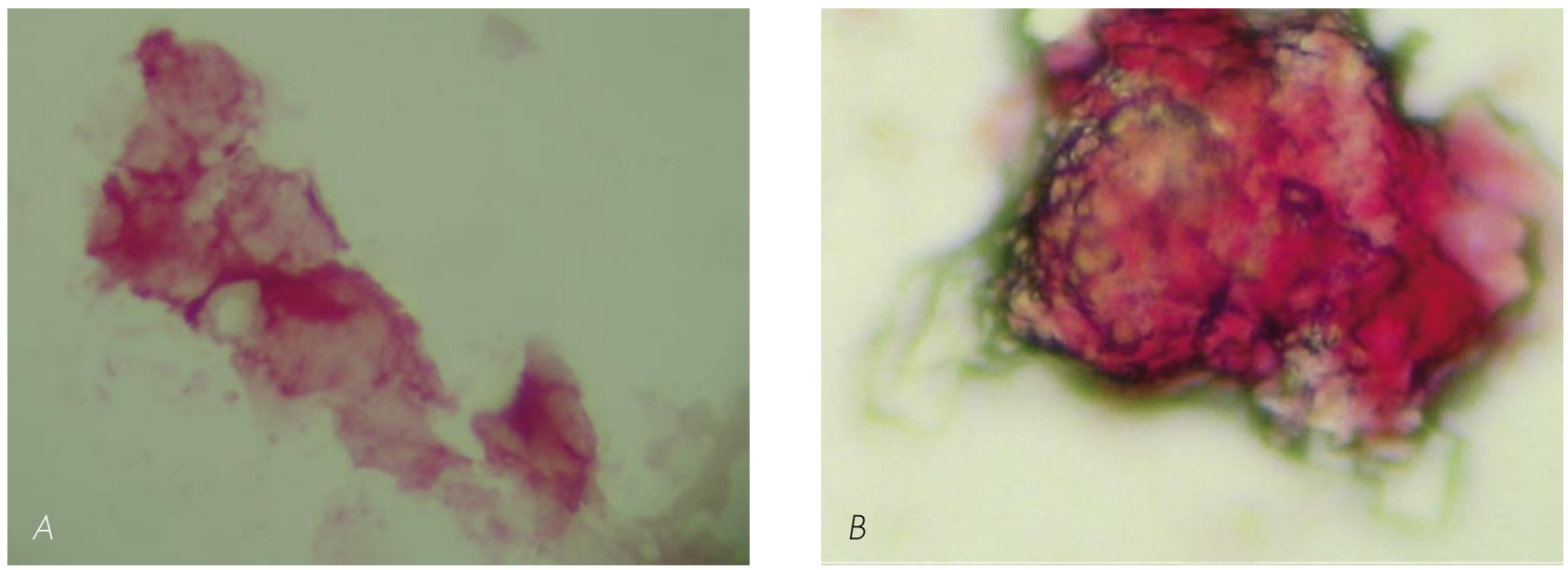

FIGURA TA y TB. Micrometástasis.

presión está disminuida o ausente en cáncer de alto grado y en macrometástasis (15). Es postulado que el CD82 actúa por la proteína quinasa C, la cual tiene un papel crucial en el ciclo celular, la migración y la invasión (15).

Presentamos un estudio sobre la detección de las $1 \stackrel{\circ}{\circ}$ CPCs y su sub-clasificación con anticuerpos monoclonales contra el supresor tumoral CD82, así como su significancia clínica en relación a la presencia de micrometástasis en la médula ósea en hombres en precirugía por cáncer prostático.

\section{MÉTODOS Y PACIENTES}

Se realizó un estudio prospectivo incluyendo todos los pacientes diagnosticados de cáncer de próstata durante el 2008 en el Hospital de Carabineros de Chile, el Instituto de BioOncología e INRAD. Se incluyeron un total de 77 pacientes con cáncer prostático confirmado por biopsia, así como 10 mujeres que formaron el grupo control. En cada individuo se registró la edad, APE total en sangre, estadio, índice de Gleason y cintigrama óseo.

Tras la recogida de todos los consentimientos informados, se obtuvieron las muestras de sangre y de médula ósea por aspiración y biopsia necesarias para el estudio.

\section{Preparación de la Muestra:}

$8 \mathrm{ml}$ de sangre venosa fueron obtenidos de la vena cubital, usando aguja de $21 G$ y 2 tubos con anticoagulante EDTA (Beckinson-Vacutainer). Se obtuvo la muestra de médula ósea desde la cresta ilíaca postero superior, usando como sedación $5 \mathrm{mg}$ de midazolam e.v. y lidocaína al $2 \%$ como anestesia local.
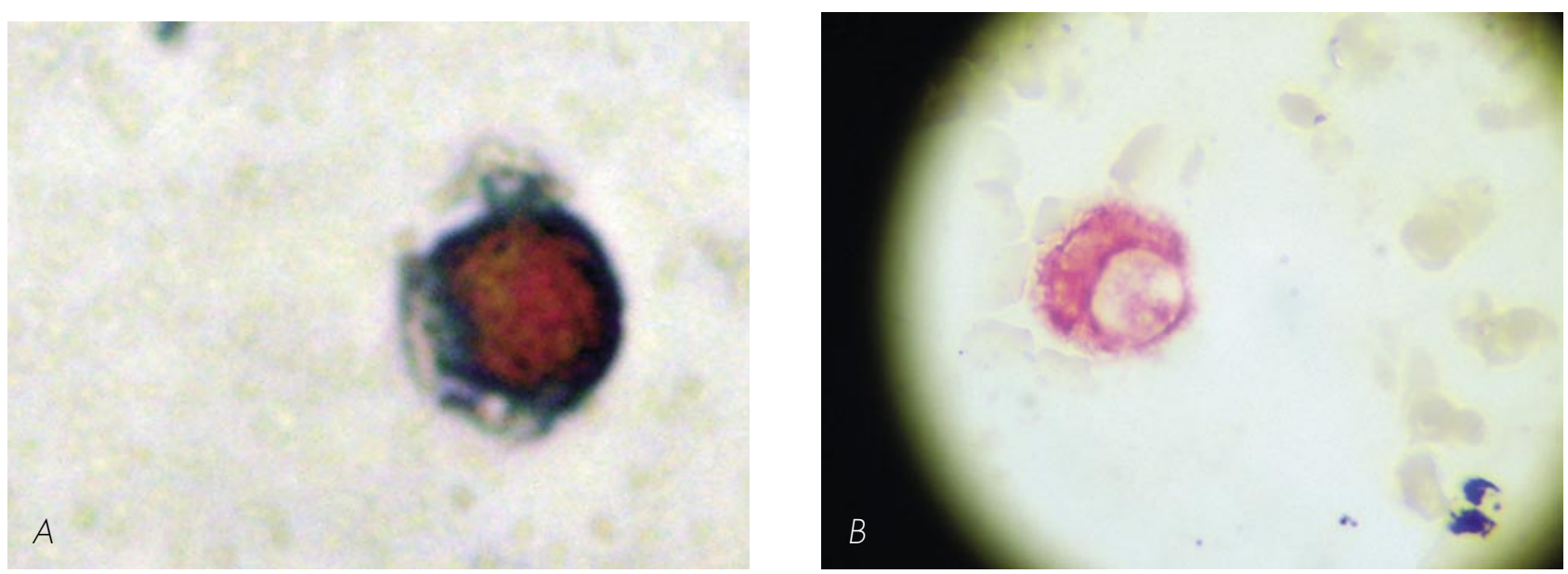

FIGURA 2A). 1.3: CPC CD82 (3+). - FIGURA 2B). 1.4: CPC CD82 (-) 
Se aspiraron un total de $4 \mathrm{ml}$ de médula ósea de cada paciente se almacenaron en tubos con anticoagulante EDTA. Se utilizó la biopsia de médula ósea para preparar 3 láminas tipo impronta.

\section{Separación de las células mono-nucleares:}

Las células mono-nucleares fueron obtenidas a partir de las muestras de sangre y médula ósea utilizando centrifugación diferencial con Histopaque 1.077 (Sigma - Aldrich), según las instrucciones del fabricante. $25 \mu \mathrm{l}$ de la suspensión obtenida fueron utilizados para preparar cada lámina (porta objeto sialinizado - DAKO EEUU). Las láminas, incluyendo las improntas, fueron secadas con aire y fijadas con solución $70 \%$ etanol, $5 \%$ formol y $25 \%$ de solución salina tamponada con fosfato $\mathrm{pH} 7.2$ por 5 minutos.

\section{Inmunocitoquímica:}

Se utilizaron anticuerpos monoclonales contra APE clon 28A4 (Novocastro Laboratory, UK) en una concentración de $25 \mu$ para detectar células prostáticas. La reacción se desarrolló con sistema de fosfatasa alcalina antifosfatasa alcalina (LSAB2 DAKO EEUU) con neofuscina como cromógena y levamisole (DAKO - EEUU) como inhibidor de fosfatasa alcalina endógeno según las instrucciones del fabricante.

\section{Anti-CD82:}

Las muestras positivas para APE tuvieron una segunda estadio de procesamiento para la detección de CD82 clon 5B5 (Novocastro Laboratory, Reino Unido) con una dilución de 1:50. Tras una incubación con inhibidor de peroxidasa (DAKO, EEUU), se desarrolló la reacción con un sistema basado en peroxidasa (LSAB2, DAKO, EEUU) con Vector VIP (Laboratory Vector, EEUU) como cromogen, según las instrucciones del fabricante.

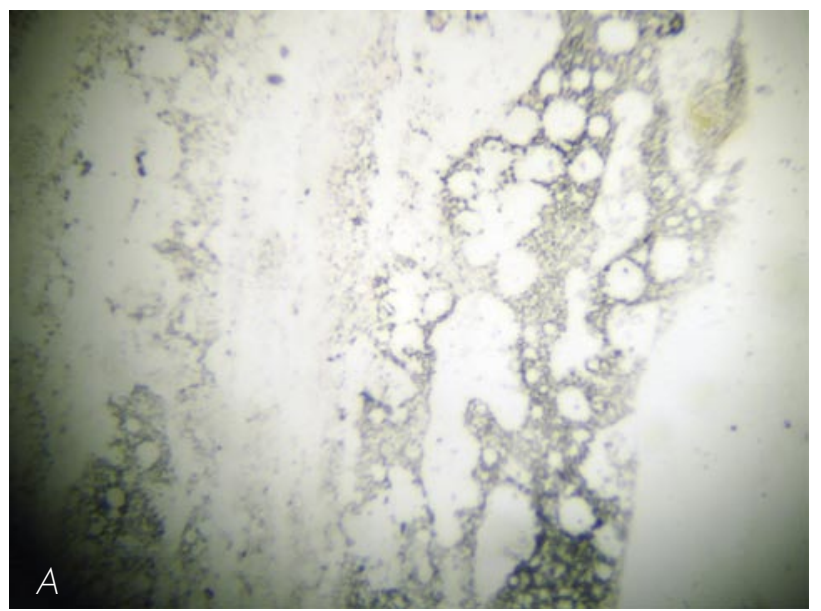

La intensidad de la expresión de CD82 fue clasificado de $\mathrm{O}$ a +++ utilizando el mismo sistema de puntaje del HercepTest por cada muestra para dar un puntaje promedio entre $\mathrm{O}$ a 3 .

La definición de una CPC o células cancerosas aisladas en la médula ósea (CCA) fue según los criterios de ISHAGE 1999 (16). Una micrometástasis fue definida como un micro-fragmento (grumo) positivo de células que expresan APE (17).

\section{4) Análisis estadística:}

$\mathrm{Se}$ usaron técnicas estadísticas descriptivas para las variables demográficas, la prueba de T-Student para las diferencias entre la edad y APE y la prueba de Chi-cuadrado para las diferencias en al frecuencia de células positivas entre los diferentes subgrupos.

Se consideró un error alfa de 0,05, un error beta de 0,20 y $p<0,05$ como significativo.

El análisis se realizó con el programa Epilnfo® para Windows $98 \AA$.

\section{5) Consideraciones éticas:}

El estudio fue desarrollado en conformidad de los principios de la declaración de Helsinki y tuvo la aprobación del Comité Ética local.

\section{RESULTADOS}

Durante el periodo comprendido en la investigación, 77 pacientes cumplieron con los criterios de inclusión para el estudio, con una edad promedio de $68,4+/-9,7$ años y un APE sérico promedio de $11,27 \mathrm{ng} / \mathrm{ml}$ (rango 1,5-85ng/ml).

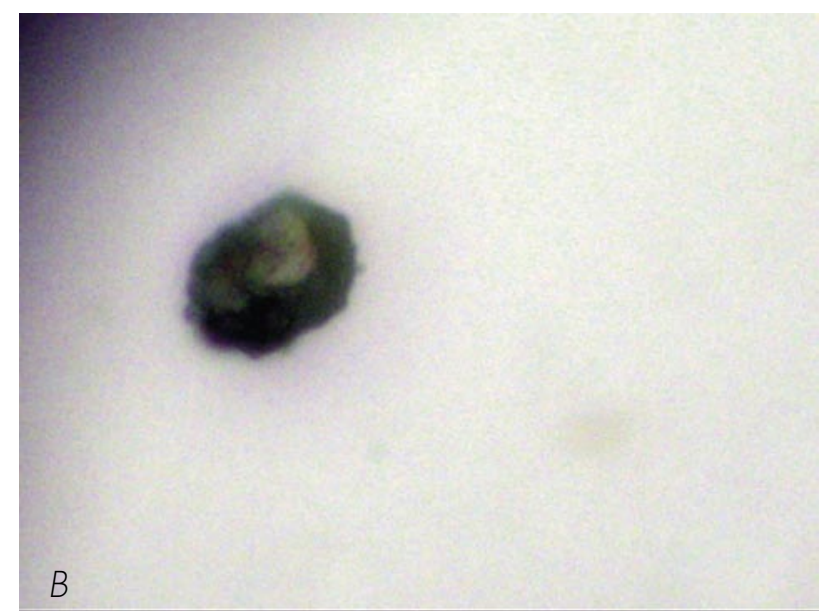


TABLA I. FRECUENCIA DE LA DETECCIÓN DE $1^{\circ}$ CPCS SEGÚN EL ESTADIO PATOLÓGICO.

\begin{tabular}{|c|c|c|c|}
\hline & $1^{\circ} \mathrm{CPC}$ positiva & $1^{\circ} \mathrm{CPC}$ negativa & Total \\
\hline Estadio 1 & 0 & 4 & 42 \\
\hline Estadio 2 & 29 & 13 & 31 \\
\hline Estadio 3 & 29 & 2 & 77 \\
\hline Total & 58 & 19 & 4 \\
\hline
\end{tabular}

Estadio $1+2$ vs Estadio $3 p=0,002$ (Chi-cuadrado 2 colas)

Estadio 2 vs Estadio $3 p=0,02$ (Chi-cuadrado 2 colas)

De los 77 pacientes, en $58(75,3 \%)$ se detectaron $1^{\circ} \mathrm{CPC}$ s en sangre venosa. La frecuencia según la estadio clínica y el índice de Gleason se muestra en la Tabla I y II. No existieron diferencias significativas entre los diferentes índices de Gleason, pero 4 pacientes con estadio $\mathrm{pT} 2$ tuvieron una frecuencia de CPCs menor que estadio pT3, 29/42 $(21,4 \%)$ vs $25 / 27(92,5 \%)$ respectivamente $(p<0,02$ Chi-cuadrado 2 colas).

De los 77 pacientes, $39(50,6 \%)$ tuvieron un mielograma positivo para células prostáticas y 31 $(40,3 \%)$ presentaron enfermedad micrometastasíca (Tabla III y IV). La presencia de células prostáticas en la aspiración (CCAs) y la presencia de micrometástasis se asoció con la estadio clínica, pT2 versus pT3 para CCAs $15 / 27(55,6 \%)$ versus $19 / 27(70,4 \%)$ $\mathrm{p}<0,005$ Chi-cuadrado 2 colas) y para la biopsia $10 / 42(23,4 \%)$ versus $17 / 27(63,0 \%)(p<0,001 \mathrm{Chi}$ cuadrado 2 colas) respectivamente. No hubo una diferencia significativa entre la frecuencia de detección de las CCAs y las micrometástasis en pacientes con estadio $\mathrm{pT} 2$ o $\mathrm{pT} 3,(\mathrm{p}=0,23$ y $\mathrm{p}=0,56$ Chi cuadrado 2 colas) respectivamente.

No hubo una relación entre el índice de Gleason y la presencia de células circulantes en la aspiración (Tabla IV), pero hubo una relación signifi-

TABLA II. FRECUENCIA DE LA DETECCIÓN DE $7^{\circ}$ CPCS SEGÚN EL ÍNDICE DE GLEASON.

\begin{tabular}{|c|c|c|c|}
\hline & $1^{\circ}$ CPC positiva & $1^{\circ}$ CPC negativa & Total \\
\hline Gleason 4 & 14 & 5 & 19 \\
\hline Gleason 5 & 15 & 6 & 27 \\
\hline Gleason 6 & 21 & 6 & 6 \\
\hline Gleason 7 & 4 & 2 & 4 \\
\hline Gleason 8 y 9 & 4 & 0 & 77 \\
\hline Total & 58 & 19 & 67 \\
\hline
\end{tabular}

Gleason 4 vs $5 p=0,87$ Gleason 4 vs $6 p=0,75$ Gleason 4 vs Gleason 7,8,9 p=0,71 
TABLA III. FRECUENCIA DE CÉLULAS PROSTÁTICAS DETECTADAS EN MÉDULA ÓSEA.

\begin{tabular}{|l|c|c|c|c|c|}
\hline & Mielograma positivo & Mielograma negativo & Biopsia positivo & Biopsia negativo & Total \\
\hline Estadio 1 & 2 & 2 & 0 & 4 & 4 \\
\hline Estadio 2 & 15 & 27 & 10 & 32 & 42 \\
\hline Estadio 3 & 23 & 8 & 21 & 10 & 31 \\
\hline Total & 40 & 37 & 31 & 46 & 77 \\
\hline
\end{tabular}

Estadio $1+2$ versus Estadio $3+4$ : Mielograma (aspiración) $p<0,001$ (Chi cuadrado 2 colas). Biopsia: $p<0,00005$ (Chi cuadrado 2 colas)

Estadio 2 versus Estadio 3: Mielograma p<0,005 (Chi cuadrado 2 colas), biopsia $p<0,001$ (Chi cuadrado 2 colas).

Mielograma versus biopsia: estadio $2 p=0,23$ estadio $3 p=0,56$.

cativa entre el índice de Gleason y la frecuencia de detección de las micrometástasis. Pacientes con un índice de Gleason de 4 o 5, tuvieron una frecuencia de micrometástasis significativamente menor que los pacientes con un índice de Gleason mayor (Tabla IV) (Chi-cuadrado 2 colas). En pacientes con Gleason 4, la frecuencia de micrometástasis fue menor que la frecuencia de células aisladas en la aspiración ( $p$ $<0,027$, Chi cuadrado 2 colas).
Hubo una diferencia significativa en la presencia de micrometástasis entre pacientes $C P C$ negativa y positiva, $1 / 19$ versus $30 / 58$ respectivamente ( $p<0,0003$ Chi cuadrado 2 colas).

\section{Sub-clasificación de las CPCs:}

CD82: Se encontraron CPCs positivas para CD82 en todos los pacientes con Gleason 4 (14/14 $100 \%$ ) y en solo cuatro pacientes con índice de Glea-

TABLA IV. LA FRECUENCIA DE CÉLULAS PROSTÁTICAS EN MÉDULA ÓSEA SEGÚN EL ÍNDICE DE GLEASON.

\begin{tabular}{|l|c|c|c|c|c|}
\hline & Mielograma positivo & Mielograma negativo & Biopsia positivo & Biopsia negativo & Total \\
\hline Gleason 4 & 8 & 11 & 2 & 17 & $19^{*}$ \\
\hline Gleason 5 & 8 & 13 & 4 & 17 & $21^{* *}$ \\
\hline Gleason 6 & 17 & 10 & 17 & 10 & $27^{* * *}$ \\
\hline Gleason 7 & 2 & 4 & 4 & 2 & $6^{* * *}$ \\
\hline Gleason 8+9 & 4 & 0 & 4 & 0 & $4^{* * *}$ \\
\hline Total & 40 & 37 & 31 & 46 & $77^{* * *}$ \\
\hline
\end{tabular}

Gleason 4 versus 5: Mielograma $p=0,80$ biopsia $p=0,45$

Gleason 4 versus 6: Mielogram $p=0,16$ biopsia $p<0,0005$

Gleason 4 versus 7/8/9: Mielograma $p=0,36$ biopsia $p<0,0001$

Gleason 5 versus 6: biopsia $\mathrm{p}<0,002$

Gleason 5 versus 7/8/9: biopsia $p<0,002$ (Fisher, 2 colas)

Mielograma versus biopsia: ${ }^{*} p<0,027{ }^{*} p=0,17 * * * p=N S$ 
son $5(4 / 15,25 \%)$. Todos los pacientes con índice de Gleason mayor a 5 fueron CD82 negativos. Los pacientes con CPCs CD82 positivos tuvieron una menor frecuencia de micrometástasis en comparación con los pacientes CD82 negativos ( $p<0,0005 \mathrm{Chi}$ cuadrado 2 colas Tabla V). 4/20 versus $26 / 38$ respectivamente. Cuando combina pacientes CPC negativos y CPC positivos con expresión de CD82, 5/39 fueron micrometástasis positiva versus $26 / 38$ micrometástasis negativa $(p<0,0000007$ Chi cuadrado 2 colas).

Comparando los 3 grupos para la predicción de la ausencia de micrometástasis y como consecuencia un candidato para la cirugía curativa, la ausen- cia de CPCs tuvo una sensibilidad de $94,7 \%$ y especificidad de 39,1\%; entre pacientes CPC positivos, el uso de la expresión de CD82 tuvo una sensibilidad de $80 \%$ y especificidad de $57 \%$, la combinación de CPC negativa y CPC positiva CD82 positivo tuvo una sensibilidad de $87 \%$ y especificidad de $73,9 \%$.

\section{DISCUSIÓN}

Los resultados del estudio ProTECT (Prostate Tumor, Early Cancer Test) implican que es posible al tiempo del diagnóstico de cáncer prostático predecir los pacientes negativos para micrometástasis independiente de la estadio, Índice de Gleason y APE sérico.

\section{TABLA V. COMPARACIÓN ENTRE LOS 3 GRUPOS, CPC NEGATIVA, CPC POSITIVO ACD82 NEGATIVO Y POSITIVO.}

\begin{tabular}{|c|c|c|c|c|}
\hline & CPC negativa & $\begin{array}{l}\text { CPC positiva } \\
\text { CD82 negativo }\end{array}$ & $\begin{array}{l}\text { CPC positiva } \\
\text { CD82 positivo }\end{array}$ & Total \\
\hline $\mathrm{N}^{\circ}$ pacientes & 19 & 39 & 19 & 77 \\
\hline Edad promedio & $68,9+/-5,8$ años & $64,7+/-9,9$ años & $75,3+/-8,5$ anos & $68,4+/-9,7$ años \\
\hline $\begin{array}{l}\text { APE al tiempo de la } \\
\text { biopsia }\end{array}$ & $\begin{array}{l}5,83+/-2,67 \\
\mathrm{ng} / \mathrm{ml}\end{array}$ & $\begin{array}{l}12,93+/-11,6 \\
\mathrm{ng} / \mathrm{ml}\end{array}$ & $\begin{array}{l}12,41+/-12,0 \\
\mathrm{ng} / \mathrm{ml}\end{array}$ & $\begin{array}{l}11,27+/-10,61 \\
\mathrm{ng} / \mathrm{ml}\end{array}$ \\
\hline $\begin{array}{l}\text { Estadio patológico } \\
\text { pT1 } \\
\text { pT2 } \\
\text { pT3 }\end{array}$ & $\begin{array}{l}4 \\
13 \\
2\end{array}$ & $\begin{array}{l}0 \\
16 \\
23\end{array}$ & $\begin{array}{l}0 \\
13 \\
6\end{array}$ & $\begin{array}{l}4 \\
42 \\
31\end{array}$ \\
\hline $\begin{array}{l}\text { Índice de Gleason } \\
4 \\
5 \\
6 \\
7 \\
8 \text { y } 9\end{array}$ & $\begin{array}{l}5 \\
6 \\
6 \\
2 \\
0\end{array}$ & $\begin{array}{l}0 \\
11 \\
21 \\
4 \\
4\end{array}$ & $\begin{array}{l}14 \\
4 \\
0 \\
0 \\
0\end{array}$ & $\begin{array}{l}19 \\
21 \\
27 \\
6 \\
4\end{array}$ \\
\hline $\begin{array}{l}\text { Micrometástasis } \\
\text { Positiva } \\
\text { Riesgo relativo }\end{array}$ & $\begin{array}{l}1(5,3 \%) \\
1,00\end{array}$ & $\begin{array}{l}26(66,7 \%) \\
4,80\end{array}$ & $\begin{array}{l}4(20 \%) \\
36,00\end{array}$ & $31(40,3 \%)$ \\
\hline $\begin{array}{l}\mathrm{N}^{\circ} \text { células detectada } \\
/ \mathrm{ml} \text { sangre }\end{array}$ & & $8+/-7$ células $/ \mathrm{ml}$ & $7+/-6$ células $/ \mathrm{ml}$ & $8+/-7$ células $/ \mathrm{ml}$ \\
\hline
\end{tabular}


Utilizando la sub-clasificación con anti-CD82 es posible identificar $73,9 \%$ de los pacientes sin micrometástasis y por lo tanto aquellos pacientes con más posibilidades de una cirugía curativa.

El presente estudio es el primero que logra demostrar que las CPCs expresan CD82 y que existe una relación entre su expresión, el índice de Gleason y la estadio clínica, semejante a lo informado por otros estudios que detectaron este marcador en células de tejido prostático obtenido de prostatectomías radicales $(14,15)$.

Nuestros resultados sugieren que la expresión del CD82 no es suficiente por si sola para inhibir la diseminación de las CPCs del tumor primario o desde los microfocos en tejidos distantes. La asociación negativa con la presencia de las micrometástasis óseas sugiere que el CD82 funciona para inhibir la implantación ósea.

Katz et al (18) han definido el concepto de fallo quirúrgico potencial como la presencia de márgenes quirúrgicos positivos, invasión de vesículas seminales o de ganglios linfáticos, para diferenciarlo de aquellos casos en los que el tumor es órgano-confinado. Sin embargo, esta definición no se incluye en el grupo los pacientes con una patología desfavorable indicada o invasión capsular pero con márgenes negativos. Se ha demostrado que este grupo presenta una evolución más favorable que si hubieran presentado márgenes positivos (19). En el cáncer clínicamente localizado han demostrado que los más importantes factores predictivos de extensión extra-capsular son la concentración sérica de PSA, el índice de Gleason y la estadio clínica, el PSA sérico se ha correlacionado con el volumen tumoral y como consecuencia con los márgenes positivos y la penetración capsular (20). El uso de la detección de CPCs y su sub-clasificación parece predecir de manera significativa e independiente la ausencia de micrometástasis en pacientes previo a la prostatectomía radical.

\section{CONCLUSIONES}

Proponemos que la presencia de CPCs implica un riesgo mayor de desarrollar micrometástasis, la co-expresión del CD82 as asociada con tumores de bajo grado, un riesgo disminuido del desarrollo de micrometástasis óseas. Como consecuencia, el uso de la detección de CPCs primarias y su sub-clasificación podrían ser clínicamente útiles para identificar los pacientes que se beneficiarán de una prostatectomía radical como tratamiento de primera línea. Los pacientes negativos para CPCs y ellos con CPCs CD82 positivos deberían ser considerados para ci- rugía sola, pacientes CPC positiva CD82 negativo deberían ser evaluados para la presencia de micrometástasis y la necesidad de terapia adyuvante.

El uso de análisis de sangre, es simple, no invasivo y podría ser implementado en el laboratorio de rutina. Las conclusiones preliminares del estudio ProTECT merecen estudios más amplios para confirmar los resultados.

\section{AGRADECIMENTOS}

Agradecemos el Consejo de Docencia del Hospital de Carabineros de Chile por el apoyo financiero del estudio y la señora Ana María Palazuelos de Murray por su apoyo en la redacción del manuscrito.

\section{BIBLIOGRAFÍA y LECTURAS RECOMENDADAS ( ${ }^{*}$ lectura de interés $y^{* *}$ lectura fundamental)}

1. Partin AW, OesterlingJE. The clinical usefulness of prostate specific antigen: Update. J Urol 1994; 143: 358-64.

2. Mettlin CJ, Jones GW, Murphy GP. Trends in prostate cancer care in the United States. 19741990: observations from the patient care evaluation studies of the American College of Surgeons Commission on Cancer. CA Cancer J Clin 1993; 43: 83-91.

3. Lu-Yao G, McLerran D, Wasson J, Wennberg JE. An assessment of radical pprostatectomy. Time trends, geographic variation and outcomes. The prostate patient outcomes research team. JAMA 1993; 269: 633-6.

4. Epstein JL, Pizov G, Walsh PC. Correlation of pathologic findings and progression after radical prostatectomy: facts and open questions. Eur Urol 1997; 32: 41-7.

*5. Bosch SCH, Schroeder FH. Current problems in staging and grading prostatic carcinoma with special reference to $\mathrm{T} 3$ carcinoma of the prostate. World J Urol 1986; 4:141-6.

6. Watson RB, Civantos F, Soloway MS. Positive surgical margins with radical prostatectomy: detailed pathological analysis and prognosis. Urology 1996; 48: 80-90.

7. van den Ouden D, Davidson PJRT, Hop W, Schroeder FH. Radical prostatectomy as a monotherapy for locally advanced (stage T3) prostate cancer. J Urol 1996; 151: 646-51. 
**8. Partin A, Yoo J, Carter HB, Pearson D, Chan DW, Epstein JL, Walsh PC. The use of prostate specific antigen, clinical stage and Gleason score to predict pathological stage in men with localized prostate cancer. J Urol 1993; 150: 110-4.

*9. Fleming C, Wasson JH, Albertsen PC, Barry MJ, Wennberg JE: A decision analysis of alternative treatment strategies for clinically localized prostate cancer. JAMA 1993; 269: 2.650-8.

10. Murray NP. El uso de doble-inmunomarcación para detectar células prostáticas en la circulación sanguínea (CPCs) en pacientes con cáncer prostático y las correlaciones con los parámetros clínicos. Rev Chil Urol 2006; 71: 135-40.

11. Murray NP, Badinez L. Las células prostáticas en la circulación sanguínea en pacientes con cáncer prostático expresan la proteína P504S: un estudio utilizando inmunocitoquímica. Rev Chil Urol 2008; 73: 54-7.

12. Murray NP. La expresión HER-2 en células prostáticas en la circulación sanguínea (CPCs) y el efecto del tratamiento con la supresión androgéncia en pacientes con cáncer de la próstata. Rev Chil Urol 2007; 72: 190-4.

13. Dong JT, Suzuki H, Pin SS, Bova GS, Schalken JA, Issacs WB et al. Down regulation of the KaI1 metastasis suppressor gene during the progression of human prostatic cancer infrequently involves gene mutation or allelic loss. Cancer Res 1996; 56: 4387-90.

14. Lijovic M, Somers G, Frauman AG. KAI1/CD82 protein expression in primary prostate cancer and in BPH associated with cancer. Cancer Detect Prev 2002; 26: 69-77.
*15. Jackson P, Ow K, Yardley G, Delprado W, Quinn DI, Yang JI et al. Downregulation of KAI1 mRNA in localized prostate cancer and its bony metastasis does not correlate with p53 overexpression. Prostate Cancer prostatic Dis 2003; 6: 174-81.

16. Borgen E, Naume B, Nesland JM, Kvalhein E, Beiske K, Fodsted O et al. Standardization of the immunocytochemical detection of cancer cells in BM and blood: Establishment of objective criteria for the evaluation of immunostained cells. Cytotherapy $1999 ; 5: 377-88$.

*17. Murray NP, Calaf GM, Badinez L. Presence of prostate cells in bone marrow biopsies as a sign of micrometastasis in cancer patients. Oncol Reports 2009; 21: 571-5.

18. Katz AE, De Vries GM, Benson MC, Buttyan RE, O'Toole K, Ruben MA et al. The role of the reverse transcriptase polymerase chain reaction assay for PSA in the selection of patients for radical prostatectomy. Urol Clin North Am 1996; 23: 541-9.

19. Witjes WP, Sculman CC, Debruyne FM. Preliminary results of a prospective study comparing radical prostatectomy versus radical prostatectomy associated with neoadjuvant hormonal combination therapy in T2-3 N0 M0 prostatic carcinoma. The European Study Group on Neoadjuvant Treatment of Prostate Cancer. Urol 1997; 49 (3A Suppl) 65-9.

20. Stamey TA, Villers AA, McNeal JE, Link PC, Freiha FS. Positive surgical margins at radical prostatectomy: importance of apical dissection. $\mathbf{J}$ Urol 1990; 143: 166-72. 